\title{
La mission Griaule à Kangaba (Mali)
}

Walter E. A. van Beek et Jan Jansen

\section{(2) OpenEdition}

Journals

Édition électronique

URL : http://journals.openedition.org/etudesafricaines/177

DOI : $10.4000 /$ etudesafricaines. 177

ISSN : $1777-5353$

\section{Éditeur}

Éditions de l'EHESS

\section{Édition imprimée}

Date de publication : 1 janvier 2000

Pagination : 363-376

ISBN : 978-2-7132-1356-4

ISSN : 0008-0055

\section{Référence électronique}

Walter E. A. van Beek et Jan Jansen, "La mission Griaule à Kangaba (Mali) », Cahiers d'études africaines [En ligne], 158 | 2000, mis en ligne le 12 juin 2004, consulté le 05 février 2021. URL : http:// journals.openedition.org/etudesafricaines/177 ; DOI : https://doi.org/10.4000/etudesafricaines. 177

Ce document a été généré automatiquement le 5 février 2021

(c) Cahiers d'Études africaines 


\title{
La mission Griaule à Kangaba (Mali)
}

\author{
Walter E. A. van Beek et Jan Jansen
}

« Vers 20 heures, le Commandant de Subdivision fait demander Marcel Griaule "pour une affaire très grave". Les griots ont prévenu le patriarche de Kéla qu'on voulait "prendre leur parole", autrement dit l'enregistrer sur bande magnétique et que, si cela se faisait, ils retourneraient à Kéla. Le Commandant de Subdivision a donné sa parole d'honneur que rien n'avait été pris et nous décidions de partir avec les voitures et de ne pas revenir avant le lendemain matin » (de Ganay 1995 : 149). Ici, la note suivante a été ajoutée : « Notre équipe, qui entendait étudier en profondeur une cérémonie dont on parlait tant [...] mais qu'aucun chercheur n'avait jusque-là observée, s'était offert les moyens de "fixer" sur bandes magnétiques les chants et récits des griots, ce qu'elle fit pour l'essentiel» (nous soulignons).

Plusieurs contributions ont été écrites sur les fameuses cérémonies septennales du Kamabolon à Kangaba, village situé à quatre-vingt-quinze kilomètres au sud de Bamako (Mali) et, probablement, une ancienne capitale de l'empire de Mali. Les rapports de G. Dieterlen $(1955,1959)$, suivis des publications de C. Meillassoux (1968) et G. Dieterlen (1968), sont devenus des classiques dans les études mandingues. Ces deux auteurs ont admis l'impossibilité d'enregistrer les paroles récitées dans le sanctuaire Kamabolon. Ces paroles sont considérées partout comme les plus grands secrets du Manden, conservés par la fameuse famille Diabaté de Kéla, griots-clients des anciens rois de Kangaba, les propriétaires du sanctuaire.

En 1995, plus de quatre décennies après avoir assisté, pour la première fois, à la cérémonie, Solange de Ganay, membre de l'équipe dirigée par M. Griaule, à laquelle G. Dieterlen appartenait également, nous a fourni certains de ces secrets dans une traduction des paroles qui auraient été dites dans le sanctuaire. Jusqu'à cette publication, personne ne semblait connaître l'existence de cet enregistrement. Ce texte suscite plus de questions qu'il ne fournit de réponses, bien qu'il contienne une analyse détaillée des actions de plusieurs groupes ayant assisté à la cérémonie (pp. 125-149) et une série de photos magnifiques. Nous pensons que ce texte, bien qu'il soit présenté comme une traduction mot à mot d'un enregistrement fait en 1954 à Kangaba, est en réalité le résultat d'une collaboration intensive, après 1954, avec des informateurs d'origines diverses, et ne peut donc être utilisé dans une étude sur le Manden. 
L'enregistrement mis en cause

S. de Ganay avait publié avant cette étude des travaux sur les Bambara et les Dogon.

Dans les premiers chapitres, les arguments et les informations sont surtout tirés des articles de G. Dieterlen $(1955,1959)$ et de Y. T. Cissé (1973), avec qui elle avait collaboré intensément, en 1954 ou plus tard. Les descriptions ethnographiques des actions pendant les cinq jours de la durée de la cérémonie de 1954 sont très intéressantes. Les problèmes commencent à la page 149, lorsque l'auteur annonce l'enregistrement susmentionné. Avant d'analyser le contenu du texte, nous étudierons la méthode d'enregistrement suivie par S. de Ganay afin de définir le statut du texte. En effet, la manière dont celui-ci a été fait n'est pas claire. Nous ne savons pas quelles ont été les parties « fixées » et celles qui ont été notées après l'enregistrement. Les notes sont ambiguës, voire même contradictoires. Elles suggèrent que le texte a été reconstruit des décennies après la cérémonie. Ce qui est sûr, c'est que toutes les informations ne viennent pas des « bandes magnétiques ", puisque à la page 166 la note suivante a été ajoutée à la phrase "Kanou Nyagaté a donné naissance à quatre fils » :

"Malheureusement, Tyabi [sic], notre assistant, n'a pas retenu les noms. »

Il semble que les Français, après leur départ forcé, avaient laissé leurs assistants maliens à Kangaba pour assister à la partie secrète de la cérémonie. Les deux assistants de recherche, Nyamablé Diarra et Tyâbi Coulibaly, semblent avoir passé la nuit à côté du Kamabolon, mais ont dû avoir des problèmes pour accomplir leur tâche, puisque S. de Ganay écrit : « Nos assistants Tyâbi Coulibaly et Nyamablé Diarra ayant dû partir, chassés par les gardiens du bara, le nom de ce roi n'a pu être enregistré » (p. 168, n. 125). Cette note nous fait penser que la mémoire des deux assistants a été une source importante pour la construction du texte. Selon leurs noms de famille, ils devaient être des « étrangers » d'origine bambara.

Les informateurs mis en cause

Quatre noms figurent dans les notes comme sources d'information. Une analyse de leur situation ajoute de grands problèmes à la compréhension du texte. Il s'agit des deux assistants de recherche cités plus haut, de Mansény Dyabâté qui, selon S. de Ganay, récita les paroles dans le sanctuaire et d'une personne mystérieuse appelée « l'informateur ». Puisque les deux assistants de recherche sont originaires d'une autre région, on peut douter de la valeur de leurs informations. Nous nous en tiendrons donc aux informateurs locaux.

Mansény semble être l'informateur le plus fiable car S. de Ganay le cite, dans ses remerciements, comme faisant partie des griots très célèbres à Kéla (p. 9), bien qu'aujourd'hui son nom ne soit presque jamais cité. Elle le présente comme étant le frère de « Bintou Sinè Diâbaté [sic], chef des griots de Kéla » (p. 33). Ce Bintou Sinè étant trop âgé pour faire le déplacement à Kangaba afin de réciter les paroles dans le sanctuaire, il aurait délégué son frère pour cette tâche. Il n'est pas certain que $\mathrm{S}$. de Ganay connaissait la différence entre un kumatigi, conteur officiel, et un jelikuntigi, chef des griots, ou le griot le plus âgé. En effet, Bintou Sinè et Mansény n'ont jamais été kumatigi, bien que Bintou Sinè eut été jelikuntigi dans les années 1960 (Camara 1990 ; Jansen 1995) et que Mansény le fut à la fin des années 1970.

Le kumatigi exerça ses fonctions pendant plusieurs décennies, garantissant ainsi la conservation de la tradition. Il n'y eut pas, de ce fait, beaucoup de kumatigiw (pluriel) à Kéla. La fonction est à présent occupée par Lansine Diabate, successeur de Kanku Madi, qui fut en charge entre 1960 et 1987, année de son décès. Avant lui, un certain Brehman, un père classificatoire de Lansine, exerça les fonctions de kumatigi pendant 
environ vingt ans. Ces informations sont confirmées à Kéla (Camara 1990 : 314 ; Jansen 1995 : 230) ; où l'on dit que Mansény n'a jamais été kumatigi et de ce fait on comprend mal pourquoi S. de Ganay a choisi cet informateur parmi les griots de Kéla.

«L'informateur » est la quatrième personne difficile à situer. Cet homme donne, à cinq reprises, des explications du texte (p. 158, note 94 ; p. 163, n. 112 et 114 ; p. 169, n. 130 ; p. 170, n. 135). Il est peu probable qu'il s'agisse de Mansény, car celui-ci donne les informations sous son propre nom. Par contre, il est possible que cet informateur ait été « le griot Karamoko Diawara, l'émissaire du chef de canton, qui nous a accompagnés dans tous nos déplacements » (p. 9). On peut se demander pourquoi la mission n'a pas choisi d'être accompagnée d'un Keita de Kangaba ou d'un Diabate de Kéla, car ces deux familles sont les « propriétaires » de la cérémonie du Kamabolon.

«L'informateur » pourrait également être Dyamoussa Soumano, cet homme, alors très âgé, qui rendit visite à S. de Ganay en France en 1982 pour lui fournir des informations sur les événements de 1954. Pendant plus de quarante ans il fut président de l'Union des griots du Mali, une association professionnelle des griots à Bamako, fondée au début des années 1950 (Zobel 1997). S. de Ganay le présente comme « le chef des griots de Bamako » (p. 8) et comme « le chef de tous les griots de Manden » (p. 150). Il mourut en 1992. Ainsi, éleva-t-elle le statut de Dyamoussa Soumano, originaire de Julafundo (vers Siby), dont le petit-fils, Bakari Sidiki Soumano, fut le successeur. Ce dernier devint un vrai « bricoleur ethnique » dans la société multiethnique bamakoise contemporaine. Il instaura des cérémonies « traditionnelles » et réalisa, à la radio et à la télévision, plusieurs programmes sur la tradition mandingue. Il aimait comparer des traditions différentes et discuter avec les chercheurs du Manden.

Ainsi, si l'on utilise l'information d'un ascendant d'un lignage ou d'un « bricoleur », étrangers au sujet, une critique s'impose. Par exemple, le fait que les griots de Kéla viennent saluer Djamousso Soumano (p. 150), comme « chef de tous les griots de Manden ", dans le sanctuaire avant la cérémonie, est tout à fait remarquable, parce que l'Union des griots du Mali était à l'époque une association qui venait tout juste d'être fondée.

M. Soumano et les deux assistants de recherche ne sont pas les seuls informateurs qui ne soient pas originaires de Kangaba et de Kéla (p. 23 sq.) ; deux autres informateurs viennent de Ségou (p. 28). Cela peut s'expliquer par la conviction qu'avaient les membres de l'équipe Griaule que les peuples dogon, bambara et mandingue possèdent une origine commune.

À l'exception de la date de la visite de M. Soumano en France, l'ouvrage de S. de Ganay ne contient aucune date concernant les interviews. Elle affirme avoir assisté aux cérémonies de 1954, 1975 et 1982, mais elle ne présente aucun autre enregistrement que celui de 1954.

La forme de la cérémonie et son contexte historique

En incluant des informateurs étrangers, S. de Ganay a ignoré l'origine historique de la cérémonie et sa forme actuelle. La cérémonie consiste en un renouvellement de la toiture du sanctuaire accompagné de récits secrets prononcés pendant la nuit, avant la pose du nouveau toit. Il est probable que la cérémonie soit liée à une organisation ancienne de l'armée (Seydou Camara, cité dans Jansen 2000) et qu'elle suive un cycle septennal lié à la tradition mandingue.

Dans sa forme actuelle elle est, entre autres, une manifestation de résistance de la part de l'ancienne famille royale de Kangaba. Cette famille fut envoyée en exil, par les Français, dans les années 1880 et remplacée par leurs rivaux de Figuira. Bien que les 
exilés aient pu revenir en 1909, ils ne retrouvèrent jamais leur position antérieure. En témoigne le fait que les rivaux de Figira habitent encore dans l'ancien palais du chef de canton à Kangaba. Ainsi, dans sa forme actuelle, la cérémonie exprime la tension entre les familles Keita rivales, et, bien que beaucoup de Mandingues soient d'avis que « Le Kama blon et son culte sont éternels» (p. 9), elle a évolué dans le temps (Jansen 2000). S. de Ganay cependant ne discute pas cette dimension politico-historique. Ce manque d'étude historique apparaît également en relation avec la photo la plus intéressante. À la page 32 la photo d'un sanctuaire bien restauré et entouré de quatre arbres, probablement des fromagers, est accompagnée de la légende suivante : « Le Kama blon en 1905 [?]. Cliché Capitaine Desplagnes [sic], Musée de l'homme [sic]. » La date est remarquable parce qu'elle renvoie à la période de l'exil des propriétaires actuels de la cérémonie. Dans son étude, $\mathrm{S}$. de Ganay ne donne aucune autre référence de cette photo. Aujourd'hui, il n'y a plus d'arbres autour du sanctuaire, mais les images nous montrent qu'en 1954 il y en avait encore deux ou trois. Il est possible qu'autrefois le Kamabolon ait été entouré de quatre arbres. Ce qui importe ici, c'est l'homme, Desplagnes, qui prit cette photo. Selon le résumé de son journal (Gollnhofer \& Sillans 1974, 1975), Desplagnes n'est jamais allé au sud de Bamako. Peut-être S. de Ganay a-telle eu plus d'informations concernant l'origine de ce cliché, étant donné qu'elle a collaboré intensivement avec Y. T. Cissé qui, selon Gollnhofer et Sillans, aida à l'archivage des documents de Desplagnes.

Le contenu du texte

Le texte lui-même est incroyablement court pour un récit qui dura toute une nuit, surtout si on le compare à d'autres publications sur les traditions orales. Cependant, S. de Ganay donne l'impression qu'il s'agit d'un texte transcrit mot à mot. Il ne compte que 22 pages (pp. 151-172), ce qui fait douter de l'authenticité de l'enregistrement. Le récit est souvent très court lorsqu'il s'agit d'événements extrêmement importants pour les habitants du Manden. Par exemple, à la page 169 elle écrit : « Soundyata descend de Maghan Konate. Ce dernier avait plusieurs épouses. Quelques-unes eurent des enfants, d'autres pas. » Nous pensons qu'aucun spécialiste de la tradition orale n'admettrait que ce thème puisse être traité ainsi, avec ces paroles, par un griot de Kéla.

Une bonne partie du texte ne traite pas de l'histoire de Sounjata, la spécialité des griots de Kéla, mais de la création, à neuf reprises, d'une personne qui s'appelle Arama. Ce dernier était « seul sur la terre » et « [...] pour l'égayer, pour qu'il soit joyeux dans son domaine, Dieu fit sortir de lui sa compagne. Celle-ci se nommait Ba Awa "Mère Awa". Awa signifie "l'écartement de la création", son enlargissement, le départ et le retour de la création, c'est-à-dire son mouvement » (p. 159). Cette citation peut servir de point de référence exemplaire à toute notre critique. Il est clair que ces noms sont ceux d'Adam et Ève. L'auteur présente néanmoins, avec l'aide explicite de son assistant, Tyabi Coulibaly, une étymologie mandingue d'Awa (p. 159, n. 96).

Pendant les quatre décennies de silence entre l'enregistrement et la publication aucun chercheur n'a publié de texte ressemblant à celui des neuf Adam. Bien que les paroles récitées pendant la cérémonie aient été publiées deux fois (Ly-Tall et al. 1987, enregistrement en 1979 ; Jansen et al. 1995, enregistrement en 1992), ainsi que des rapports sur les répétitions (Jansen 1999, 2000), avec pour résultat des textes presque identiques, on ne trouve ni l'histoire des neuf Adam, ni les autres récits publiés par S. de Ganay dans ces deux textes. En plus, le récit que relate S. de Ganay ne ressemble pas du tout à « la légende officielle » transcrite par J. Vidal dans les années 1920, la plus 
ancienne version que l'on possède de Kéla. Tout cela fait du texte de S. de Ganay une étrange curiosité.

L'absence de ces généalogies, typiques pour la région de Kangaba, pose encore un problème et incite à penser que le texte ne serait pas à l'origine de cette région. Ces généalogies représentent des revendications politiques, qui sont très stables dans une région (Jansen 1996). Les griots en sont très fiers; ils les récitent lors de tous les événements officiels. Cependant S. de Ganay ne les mentionne pas. Ainsi, il est remarquable que le récit qu'elle présente fasse état d'un certain Kanou Nyagate, qu'elle désigne comme étant le fils de Mamadu Kanou. À notre connaissance, le nom de Kanou Nyagate est inconnu dans le monde mandingue. S. de Ganay ajoute, dans une note : «Un vieillard n'est pas d'accord, et affirme que le premier fils de Mamadou Kanou est Kanou Simbo et que les griots ont "saboté" la "parole" et interverti l'ordre pour qu'on ne puisse savoir exactement » (p. 166). Le nom de Kanou Simbo est en effet bien connu dans le Manden comme le nom du premier fils de Mamadou Kanou. C'est certainement à cause de ses études bibliographiques extrêmement insuffisantes que S. de Ganay n'a pas remarqué cela.

Notre dernière critique concerne le travail superficiel de $\mathrm{S}$. de Ganay sur le terrain. Elle fournit de courtes interviews réalisées avec des informateurs qui donnent une explication sur la signification de la cérémonie, mais elle n'a fait aucun effort pour replacer la tradition dans son contexte social et politique. Elle donne peu d'informations sur les autres cérémonies auxquelles elle a assisté en 1975 et 1982, et ne parle guère des répétitions pendant la préparation de la cérémonie, répétitions qui sont ouvertes au public, contrairement à la cérémonie elle-même (Jansen 1999, 2000). Si elle y avait assisté, elle aurait su qu'une version embellie et détaillée de l'épopée de Sounjata fut récitée dans le sanctuaire. Cette version dure environ huit heures, mais S. de Ganay écrit que les griots de Kéla ont besoin de vingthuit nuits pour répéter le récit de leur tradition dans sa totalité (p. 35). Quelle différence entre un texte de 22 pages et une répétition qui dure huit heures !

Que s'est-il passé en 1954 ? Y avait-il un conteur étranger, autre que jelikuntigi officiel Brehman, et un récit déformé. Si tel est le cas, S. de Ganay aurait dû comparer la cérémonie de 1954 avec celles de 1975 et 1982, avec les autres textes publiés et avec les répétitions de Kéla. Cependant, nous croyons que tel n'est pas été le cas, si l'on se réfère à ce qu'elle écrit à la fin du récit : « Un vieillard, Sanaba Yamourou Keïta, nous dira plus tard que les griots de Kéla ne voulant pas que les "Blancs" sachent tout ce qu'ils ont dit cette nuit-là, ont "déformé" le récit traditionnel. Ils y ont ajouté, dit-il, beaucoup de choses concernant les musulmans, ressassé des épisodes sans intérêt afin de pouvoir parler jusqu'au matin, moment où ils doivent se taire, sans avoir expliqué la chose essentielle : la "sortie" des Noirs du Manden » (p. 172, n. 141).

Tout chercheur au Manden sait que son enregistrement sera souvent critiqué par quelqu'un qui lui déclarera qu'il vient d'enregistrer une chose sans valeur. De là, on peut ignorer les paroles de Sanaba Yamourou Keïta. Cependant S. de Ganay aurait dû leur prêter plus d'attention en comparant son récit avec d'autres publications, par exemple celle de Ly-Tall (Ly-Tall et al. 1997). Elle aurait dû savoir également, après avoir assisté à trois cérémonies, que les griots commencent leur récit par des discours sur La Mecque et le prophète Mahomet (Jansen 2000).

La mission Griaule en 1954

Dans l'anthropologie contemporaine on s'est aperçu que le contexte de la découverte des données, la construction d'un texte ethnographique, et, en tout cas, la connaissance 
de l'autre, ne peuvent pas être compris sans la compétence des chercheurs. Ici, l'étude de Solange de Ganay nous transporte dans un réseau de chercheurs formés par Marcel Griaule. Ce groupe de chercheurs, pionniers sur le terrain au Mali, avait des idées assez précises sur les caractéristiques des civilisations noires, le rôle des griots, les genres de traditions historiques. Aussi, sur le symbolisme, la notion du secret et la cosmologie, leurs idées étaient claires et dominantes, puisque neuves à l'époque.

L'ouvrage de S. de Ganay nous replace dans la situation scientifique des principaux textes ethnographiques que M. Griaule et son équipe ont produits sur la civilisation dogon. Bien que leurs premières publications soient intéressantes et fondamentales, une ethnographie naîtra, après 1948, avec les mêmes caractéristiques basées sur la tradition historique. L'image de la culture dogon change radicalement : les récits de Ogotemelli et Ambara, mentionnés plus haut, décrivent une civilisation centrée autour du mythe de la création, avec une connaissance élaborée de la numérologie, et une science de la cosmologie complexe, symbolique et surtout secrète. Cette image des Dogon ne correspond pas du tout aux descriptions faites auparavant (Paulme 1940), ni aux résultats des études successives (Bouju 1984 ; van Beek 1991, 1994). Ainsi, comparée à l'ethnographie africaniste, cette ethnographie griaulienne était une anomalie (Tait 1950 ; Douglas 1967, 1968).

Les textes reproduits par M. Griaule ont les mêmes caractéristiques que ceux de S. de Ganay : deux textes sur la création, avec beaucoup d'éléments venant des grandes traditions (van Beek 1991). Jamais les auteurs ne remettent en cause la provenance des thèmes et des noms. S. de Ganay (p. 155, n. 79) indique que « les Bambara ne disent pas Arama, mais Adama ». Cette note est presque la même que celle de M. Griaule dans Masques dogon (1938:46), où il note les noms alternatifs des premiers ancêtres dogon " Adama » et « Hawa »! Le refus d'accepter une provenance « non authentique » de certaines parties des textes est une des caractéristiques du projet de recherche de M. Griaule.

D'autres éléments, permettent de rapprocher le texte du sanctuaire Kama Blon du projet griaulien. Il existe partout en Afrique occidentale, et ailleurs, des mythes, des récits de fondation de royaume, de village, des formes de cultures. Le mythe de fondation s'exprime par la formule : «On est venu de... » On trouve très peu de récits de création ex nihilo. Or, l'équipe de M. Griaule trouvait des mythes de création partout. En tout cas dans la période où la cérémonie de Kamabolon se déroulait, c'est-à-dire en 1952, cette équipe était en train de travailler sur des discours du pays dogon qui aboutiraient au Renard pâle (Griaule \& Dieterlen 1965), un livre rempli de mythes de création, de numérologies et d'interprétations des dessins comme le livre S. de Ganay (van Beek 1991).

Faisons un retour en arrière pour comparer le récit de $\mathrm{S}$. de Ganay et d'autres textes recueillis par l'équipe de M. Griaule. Quelques ressemblances sont évidentes. D'abord, le texte a beaucoup de liens avec les récits de création qui font partie des grandes traditions, chrétiennes et musulmanes. La création émane d'un seul dieu, le créateur, et se base sur la cosmologie, la terre, le vent, le feu et l'eau constituant les quatre éléments principaux. Une création aussi qui se décompose en périodes de mille ans, avec l'homme (Arama) comme noyau et fondement du monde : « La création était faite pour lui et en même temps, il avait toute la responsabilité du monde » (p. 155). Il s'agit d'une création avec un esprit qui est le vent (le souffle de Dieu) et la lumière. Guère déguisé, on reconnaît Adam : « Pour qu'il soit joyeux dans son domaine, Dieu fit sortir de lui sa compagne » (supra) Ève ! Plus loin Caïn tue Abel, Noé et Abraham font leur 
apparition et enfin l'histoire de Mohammed s'introduit dans le récit de la création. Incontestablement, le texte est un bricolage des notions coraniques et bibliques, mélangées avec des éléments d'origine mandingue. Un bricolage créatif, bien sûr, qui exigeait une connaissance scientifique de la part des informateurs ou assistants dont disposait l'équipe de S. de Ganay. Un tel bricolage n'est pas surprenant, ni inconnu. La tendance à relier les traditions locales aux grands récits « du livre » est assez courante. La façon dont les ancêtres de Sunjata ont été inscrits dans la préhistoire de l'islam est répandue partout en pays mandingue, ainsi que dans la région de Kangaba. On ne trouve les mêmes thèmes dans les textes dogon recueillis par l'équipe de M. Griaule dans la région de Sangha; les fameux textes de création d'Ogotemelli (Griaule 1948) et Ambara (Griaule \& Dieterlen 1965) puisent aux sources coraniques et bibliques (van Beek 1991 ; Clifford 1988 ; Lettens 1971).

Une deuxième tendance, divergente des autres traditions mandingues, est l'importance attribuée à la numérologie. Partout dans le texte des chiffres symboles sont évoqués : les chiffres 9 (Adam), 7 (sept cieux et sept terres), 3 (nombre masculin), 4 (nombre féminin, aussi quatre éléments principaux, quatre points cardinaux), 12 villages du Manden. Pendant la cérémonie on ' fait 7 fois le tour, en chantant 3 fois, avec un tambour qui est frappé 4 fois (p. 167). Sunjata est né après une grossesse de 7 ans. Le chiffre 34 figure également dans la création. Bien que des chiffres symboles ne soient pas étrangers aux civilisations mandingues (comme 3, masculin, 4, féminin), un tel système de numérologie est inconnu.

L'étude ethnographique entreprise par S. de Ganay reflète les présupposés de M. Griaule (Clifford 1988). Pour lui, la recherche sur le terrain est semblable à une campagne « militaire » : l'ethnographie est un travail d'équipe comprenant plusieurs spécialistes des divers aspects des groupes humains. La civilisation étrangère est vue comme une forteresse qui doit être franchie par une équipe de spécialistes. Dans ce projet on parle de l'ingérence dans la vie de l'autre. L'étranger, Dogon ou Mandingue, est l'adversaire puisqu'il essaie de garder ses informations, d'empêcher l'ethnographe d'avoir accès aux données les plus intéressantes et surtout de sauvegarder les secrets tribaux. L'ethnographe doit être rusé, insistant et proche du pouvoir politique, chose facile dans l'Afrique occidentale coloniale. À ce propos, G. Dieterlen écrit un rapport destiné à l'administration coloniale : « Nous souhaitons que ce témoignage, qui a une valeur politique [Dieterlen souligne !] indéniable retienne l'attention des pouvoirs publics $»^{1}$.

Ainsi, l'équipe de M. Griaule voit les symboles comme des codes que l'on doit décrypter (Sperber 1975), afin d'aboutir aux secrets gardés. De ce point de vue, on peut les concevoir comme des secrets militaires : une information cachée, essentielle, connue d'une minorité de la population étudiée, qui n'est divulguée qu'aux gens qui doivent la connaître. Les secrets ont donc un contenu spécifique bien déterminé et abordable ; ils deviennent une chose à découvrir.

Cette attitude peut être observée dans la recherche sur les Dogon, conduite par S. de Ganay. Les paroles citées au début de notre commentaire sont claires : l'équipe vient avec des voitures, accompagnée du commandant de subdivision, affichant force et pouvoir. On essaye de découvrir des données, par des enregistrements sonores, ce qui, d'ailleurs, était interdit. Aussi, l'équipe assistant à la cérémonie de Kamabolon se limitait à la cérémonie elle-même, sans tenir compte des relations entre les griots et les autres familles. 
Les relations entre « le projet dogon » et « le projet kamabolon » sont étroites et claires. En plus des similitudes mentionnées plus haut, le texte présente également des références directes aux Dogon. Par exemple, p. 193, le texte « explique » une des significations des peintures sur le bâtiment : « Sigi loolo, "étoile du Sigui", c'est-à-dire Sirius. » Non seulement les « connaissances » des Dogon sur l'étoile de Sirius sont évoquées, mais aussi les rites dogon du sigui sont également attribués aux Mandingues. Sans aucune discussion, les Dogon sont considérés comme les véritables gardiens des secrets du Manden et placés dans une chronologie mythique.

*

Parmi une série de textes recueillis dans ceux des griots de Kéla, un nouveau texte apparaît et est présenté comme relativement ancien. La notion de la création (ex nihilo !) est introduite, avec beaucoup d'allusions coraniques et bibliques, insistant sur une numérologie insoupçonnée. Comparé avec les autres enregistrements faits au Manden, dans la période 1954-1996, ce nouveau texte est une anomalie. Nous avons essayé de comprendre le mécanisme de sa parution et de sa construction.

Le " projet Griaule », en son temps, a fourni des textes intéressants sur lesquels on peut toujours s'appuyer pour faire des études plus approfondies. Mais il a également produit des textes suspects qu'on ne peut utiliser dans la recherche contemporaine, ce qui donne lieu à quelques anomalies comme, par exemple, la publication de textes, plusieurs années après leur énonciation alors que des chercheurs les auraient déjà réfutés. Au temps de M. Griaule, la production de ce genre de textes était compréhensible ; à présent elle ne l'est plus. Le manque de références à des recherches plus récentes comme celles menées par Ly-Tall (Ly-Tall et al. 1987), lesquelles ont donné des résultats tout à fait divergents, constitue un bel exemple. S. de Ganay nous a fourni un document historique fascinant, pas tant pour l'étude du Kamabolon, ni pour celle de la culture mandingue, mais pour l'histoire de l'ethnographie griaulienne et de l'empreinte qu'elle a laissée sur une époque révolue de l'ethnographie du Mali. * À propos de : Solange de Ganay, Le sanctuaire kamablon de Kangaba. Histoire, mythes, peintures pariétales et cérémonies septennales, Paris, Éditions nouvelles du Sud, 1995.

\section{BIBLIOGRAPHIE}

Beek, W. E. A. van

1991 « Dogon Restudied. A Field Evaluation of the Work of Marcel Griaule », Current Anthropology, 32 (2) : 139-165.

1994 « The Innocent Sorcerer. Coping with Evil in Two African Societies, Kapsiki and Dogon ", in T. Blakely, W. E. A. van Beek \& D. L. Thomson, eds, African Religion :

Experience and Expression, London, James Currey : 196-228.

Bouju, J.

1984 Graine de l'homme, enfant du mil, Paris, Société d'ethnographie.

Camara, S.

1990 La tradition orale en question : conservation et transmission des traditions historiques au Manden : le centre de Kela et l'histoire de Mininjan, thèse de doctorat, Paris, EHESS. 
Cissé, Y. T.

1973 « Signes graphiques, représentations, concepts et tests relatifs à la personne chez les Malinke et les Bambara du Mali », Contribution au colloque international, 11-17 octobre 1971, La notion de personne en Afrique noire, Paris, Éditions du CNRS : 131-179. Clifford, J. 1988 « Power and Dialogue in Ethnography. Marcel Griaule's Initiation », Culture as a Predicament, Madison, University of Winconsin Press.

Dieterlen, G.

1955, 1959 « Mythe et organisation sociale au Soudan français », Journal de la Société des Africanistes, XXV : 38-76 ; XXIX (1) : 119-138.

1968 « Note complémentaire sur le sanctuaire de Kaaba », Journal de la Société des

Africanistes, XXXVIII (2) : 185-188.

Douglas, $M$.

1967 « If the Dogon... ", Cahiers d'Études africaines, VII (4), 28 : 659-672.

1968 « Dogon Culture. Profane and Arcane », Africa, 38 : 16-24.

Gollnhofer, O. \& Sillans, R.

1974, 1975 « Archives de la Société des Africanistes : documents Desplagnes », Journal de la Société des Africanistes, XLIV (1) : 189-197 ; XLV (1-2) : 201-215.

Griaule, M.

1938 Masques dogon, Paris, Institut d'ethnologie.

1948 Dieu d'eau : entretiens avec Ogotemmêli, Paris, Éditions du Chêne.

Griaule, M. \& Dieterlen, G.

1965 Le renard pâle, I : Le mythe cosmogonique ; fasc. 1 : La création du monde, Paris, Institut d'ethnologie.

Jansen, J.

1995 De Draaiende Put. Een studie naar de relatie tussen het Sunjata-epos en de samenleving in de Haut-Niger (Mali), Leiden, Research School Center of Non-Western Studies.

1996 « The Younger Brother and the Stranger : In Search of a Status Discourse for Mande ", Cahiers d'Études africaines, XXXVI (4), 144 : 659-688.

1998 « Hot Issues. The 1997 Kamabolon Ceremony in Kangaba (Mali) », International Journal of African Historical Studies, 31 (2) : 253-278.

1999 " An Ethnography of the Sunjata Epic in Kela », in R. A. Austen, ed., In Search of Sunjata : the Mande Epic as History. Literature and Performance, Bloomington, Indiana University Press : 297-312.

2000 " The Sunjata Epic. The Ultimate Version », Research in African Literatures, 30. Jansen, J., Duintjer, E. \& Tamboura, B.

1995 L'épopée de Sunjata, d'après Lansine Diabate de Kela, Leiden, Research School Center of Non-Western Studies.

Lettens, D.

1971 Mystagogie et mystification. Évaluation de l'oeuvre de Marcel Griaule, Bujumbara, Presses Lavigerie.

Ly-Tall, M., Camara, S. \& Dioura, B.

1987 L'histoire du Mandé d'après Jeli Kanku Madi Jabaté de Kéla, Paris, Association SCOA pour la recherche en Afrique noire.

Meillassoux, C.

1968 "Les cérémonies septennales du Kamablon de Kaaba », Journal de la Société des

Africanistes, 38 : 173-182.

Paulme, D. 
1940 Organisation sociale des Dogon (Soudan français), Paris, Les Éditions Domat-

Montchrestien.

Sperber, D.

1975 Rethinking Symbolism, Cambridge, Cambridge University Press.

Tait, D.

1950 « An Analytical Commentary on the Social Structure of the Dogon », Africa, 20 :

175-199.

Vidal, J.

1924 « La légende officielle de Soundiata, fondateur de l'empire manding », Bulletin du comité d'études historiques et scientifiques de l'Afrique occidentale française, 7 : 317-328.

Zobel, C.

1997 Das Gewicht der Rede ; kulturelle Reinterpretation, Geschichte und Vermittlung bei den Mande Westafrikas, Frankfurt, Peter Lang Verlag.

\section{NOTES}

1. Archives nationales, section Outre-Mer, FM 64 ; Affaires politiques, Rapports politiques, Soudan. Carton 2198, dossier 11, administration générale 1947-1957, lettre de février 1955.

\section{RÉSUMÉS}

Le livre de Solange de Ganay est un document historique fascinant, non pas tant pour l'étude du Kamabolon, ni pour celle de la culture mandingue, mais important pour l'histoire de l'ethnographie griaulienne laquelle a marqué l'ancienne ethnographie du Mali. Cette ethnographie décrite par S. de Ganay reflète le projet ethnographique de Marcel Griaule, pour qui la recherche sur le terrain est vue comme une campagne " militaire » : l'ethnographie est faite en équipe, avec des spécialistes de divers domaines. Concrètement, l'équipe de M. Griaule voit des symboles comme une cryptographie: on doit les déchiffrer, "trouver le code", afin d'aboutir aux secrets gardés par une minorité de la population étudiée. Parmi les textes sur l'histoire du Manden, recueillis dans la période 1954-1996, le texte de S. de Ganay est une anomalie. Cet article essaie d'en comprendre la création et la construction pour mettre en question la méthode de recherche et la connaissance des informateurs.

The Griaule Expedition to Kangaba (Mali). -- Solange de Ganay's book is a fascinating historical document not for the study of Kamabolon or Manding culture but for the history of Marcel Griaule's field work, which has left its marks on the ethnology of Mali. The kind of ethnological study described by de Ganay reflects Griaule's ethnological project. For him, field work was like a "military" campaign to be conducted by a team with specialists in various fields. The Griaule team saw symbols as a cryptography to be "decoded" so as to discover the secrets kept by a minority of the population. Among the texts on Manden history collected between 1954 and 1996, de Ganay's is an anomaly. How to understand its creation and construction so as to question its research methods and informers' knowledge? 
INDEX

Mots-clés : ethnographie, ethnology, cérémonies, Kamabolon, Manding traditions, Marcel Griaule expedition, mission Griaule, tradition mandingue 